\title{
Reduction and Nonlinear Controllability of Symmetric Distributed Systems with Robotic Applications
}

\author{
M. Brett McMickell \\ Bill Goodwine \\ mmcmicke@nd.edu \\ bill@controls.ame.nd.edu \\ Aerospace and Mechanical Engineering \\ University of Notre Dame \\ Notre Dame, Indiana 46556
}

\begin{abstract}
The purpose of this paper is to develop methods to reduce the complexity of nonlinear distributed systems by using symmetry propers within the system. A method for contracting and expanding controllable nonlinear systems is developed which maintains the controllability of the original system. In fact, it is shown that an entire equivalence class of symmetric nonlinear distributed control systems can be determined by checking the controllability of only one of its members. A group of mobile robots is used to demonstrate the utility of methods presented.
\end{abstract}

\section{Introduction}

This paper considers nonlinear controllability of distributed systems, which is useful to analyze large scale cooperating robotic systems. While this paper is limited to controllability, the overall purpose of this research is to develop provable methods to "reduce" the order of complexity of large scale robotic systems by exploiting symmetry within the system. These methods will be useful for robotics engineers because reduced order models are easier to consider analytically and also are computationally faster so that computationally intensive control methodologies are more easily implemented.

The main result is a proposition which can be used to determine controllability of large scale symmetric distributed systems by checking (via Chow's theorem [2]) controllability of a much smaller scale, reduced order system. The result shows that controllability of an entire equivalence class of symmetric control systems can be determined by checking the controllability of only one of its members. The use of the proposition is illustrated with a cooperating robots example.

For simplicity of presentation, this paper is limited to driftless systems, i.e., systems of the form

$$
\Sigma: \quad \dot{x}=g_{1}(x) u_{1}+\cdots+g_{n}(x) u_{n},
$$

where the $g_{i}(x)$ are smooth analytic vector fields defined on the configuration space of the system and the $u_{i}$ are control inputs. However, there are no apparent difficulties with extending the approach to systems with drift (utilizing the results from [10]).

For example, consider large scale, but structurally

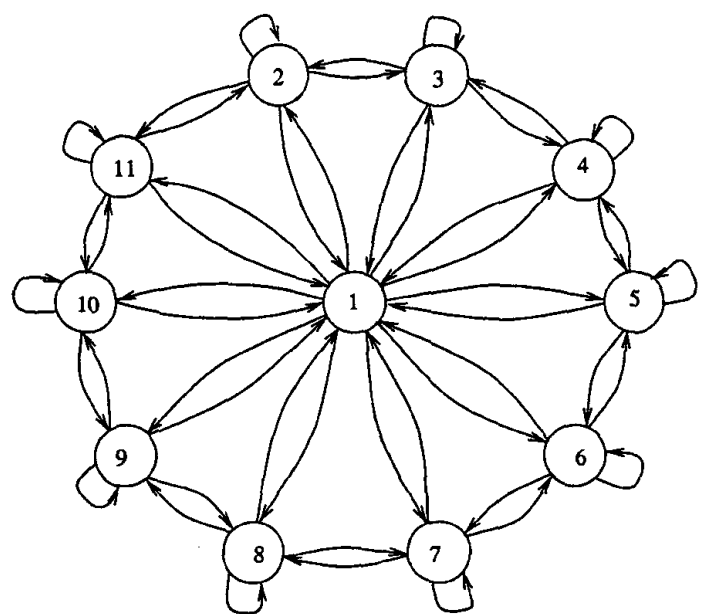

Figure 1. Ten node symmetric system

simple cooperating robotic system, with 11 robots, systematically illustrated in Figure 1 . The vertices, $V_{i}$, in the graph structure represent individual robots in the system, which mathematically represent individual configuration sub-manifolds of the entire configuration manifold of the robotic system. Furthermore, associated with each robot is a set of control inputs. The edges of the graph structure represent vector fields where an edge connecting $V_{i}$ to $V_{j}$ represents a vector field on $T V_{j}$ that is a function of the states in $V_{i}$ and $V_{j}$. These edges mathematically represent the interaction among the robots. The system is symmetric if it remains invariant when one or more robots are interchanged. A more formal description of the relationship between a graph representation and the usual differential equation representation is developed in Section 2.

Many efforts have been directed toward "reduction" of mechanical systems [1], [4], [5], [6], [7], [8] and control systems [15], [14]. However, these results are directed toward cases where there are Lie group symmetries. In contrast, this paper considers discrete symmetries. A similar approach was considered by Tanaka [11], [12], [13]; however, those results were 
limited to linear controllability, as opposed to the full nonlinear controllability considered in this paper. Furthermore, the main results in those papers were not addressing a constructive "reduction" problem, but rather determining the degree of fault tolerance of linear symmetric systems.

The remainder of this paper is organized as follows. Section 2 describes the representation of distributed systems, defines symmetries for such systems and defines equivalence classes of systems. Section 3 presents the main result, which is a nonlinear controllability test for equivalence classes of symmetric distributed control systems. Section 4 presents a simple robotics example of the use of the result from Section 3 , and Section 5 presents conclusions and outlines current and future efforts to extend the results in this paper.

\section{Symmetric Distributed Systems}

A graph theoretical approach provides a compact means to represent the topological and algebraic structure of large interconnected distributed systems. In graph theory, a digraph is a graph with directional connections between nodes or vertices. In this paper, we use digraphs to represent nonlinear control and cooperating robotic systems.

Formally, we define the digraph of a nonlinear control system $\Sigma$, written as $\mathcal{G}_{\Sigma}$, to be the pair $(\mathbf{V}, \mathbf{E})$ consisting of a set of vertices denoted by $\mathbf{V}$ and the set of ordered pairs of elements of $\mathbf{V}$, denoted by $\mathbf{E}$. A vertex, $V_{i} \in \mathrm{V}$, is a submanifold of the configuration manifold consisting of $i_{r}$ states, $\mathbf{x}_{\mathbf{i}}=\left\{x_{i_{1}}, \ldots, x_{i_{r}}\right\}^{T}$, such that the complete configuration space, $\mathcal{M}$, is the Cartesian product of all vertices given by

$$
\mathcal{M}=V_{1} \times V_{2} \times \cdots \times V_{n}=\prod_{i=1}^{n} V_{i} .
$$

An edge, $E_{i, j} \in \mathbf{E}$, also commonly referred to as an arc, represents a vector field on the tangent space of the end-point vertex $V_{j}$ which is a function of the states on $V_{i}$ and $V_{j}$ i.e.,

$$
E_{i, j}: V_{i} \times V_{j} \mapsto T V_{j}
$$

Figure 1 shows a a digraph consisting of 11 vertices and 50 edges. Each vertex has a self-referencing edge i.e., an edge with itself as the initial and final point (not illustrated for $V_{1}$ ). Since it is common for the vector fields of a subsystem to depend on states contained within its own subsystem, we will drop the selfreferencing connections for convenience and assume that any vertex can have a self-referencing edge.

In order to develop mappings between digraphs, we need to use some tools from group theory. A $G$-set is a set where there exists a function $\alpha: G \times X \mapsto X$ (called an action) such that $\alpha(1, x)=x, \forall x \in X$ and

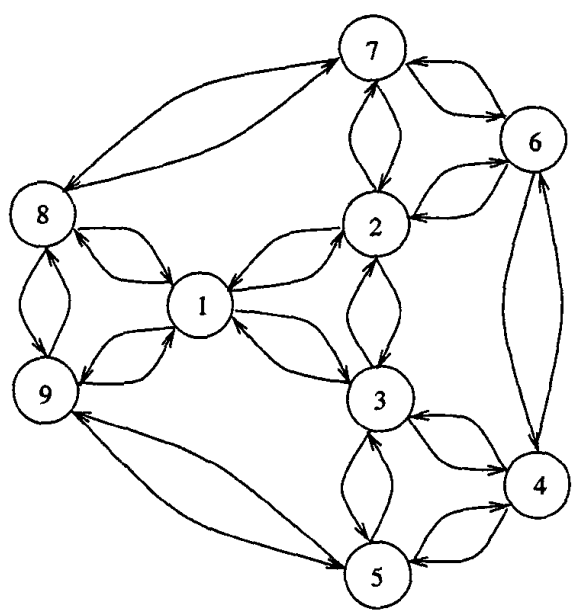

Figure 2. Complicated digraph structure.

$\alpha(\rho, \alpha(\nu, x))=\alpha(\rho \nu, x), \forall \rho, \nu \in G$ and $x \in X$. We will typically abbreviate $\alpha(\rho, x)$ by $\rho x$. A $G$-set can be partitioned into equivalence classes using $G$-orbits.

DEFINITION 2.1 If $X$ is a $G$-set and $x \in X$, then the G-Orbit of $x$ is

$$
O(x)=\{\rho x: \rho \in G\} .
$$

It is common to refer to a $G$-orbit as simply an orbit. In this paper, orbits are used to partition the system into nodes that can be interchanged and those that cannot. A fixed orbit is the set of vertices or subsystems that are unaffected by the symmetry group actions and the non-fixed orbits are the symmetry orbits.

We consider systems that have at most two cycles in the symmetry orbit. A cycle of length $\zeta, \zeta \geq 3$, is a set of $\zeta+1$ ordered vertices in which all the vertices and edges are different and the first and last vertex are the same. For the system in Figure 1, a clockwise cycle is $C_{1}=\left\{V_{2}, V_{3}, \ldots, V_{11}, V_{2}\right\}$ and a counterclockwise cycle is $C_{2}=\left\{V_{2}, V_{11}, V_{10}, \ldots, V_{3}, V_{2}\right\}$. Even with this restriction, a broad class of systems can be considered. Many very complicated digraphs can be reduced to a digraph with one cycle by combining multiple vertices into one vertex. Simple "ring like" systems, as in Figure 1 obviously satisfies this restriction, but so do a broad range of other more complex digraphs, such as the one illustrated in Figure 2, which shows that groups of vertices, such as $V_{3}, V_{4}$ and $V_{5}$ can be considered as an individual vertex which form the appropriate cycle structure.

In this paper, we restrict our attention to systems which are symmetric with respect to the symmetry group, $S_{m}$, which is the group of degree $m$ of the $m$ ! 
permutations of a set containing $m$ elements. An element of the symmetry group, $\rho$, acts on a vertex, $V_{i}$, by interchanging the elements of $V_{i}$ with elements of another vertex $V_{\rho(i)}$, i.e., $\rho V_{i}=V_{\rho(i)}$. Applying the symmetry group operator on a distributed nonlinear system is defined as follows.

DEFINITION 2.2 A nonlinear system in the form of Equation 1 is said to be symmetric if for all $\rho \in S_{m}$ such that $\rho$ interchanges vertices $V_{i}$ and $V_{j}$,

$$
\begin{gathered}
\rho_{*} E_{i, k}=E_{j, k}, \quad \forall k \notin C \\
\rho_{*} E_{i, l_{i} \pm 1}=E_{j, l_{j} \pm 1},
\end{gathered}
$$

where $C$ is the set of cycles, if any, (clockwise and counterclockwise) through the symmetry orbit, $E_{i, l_{i} \pm 1}$ denotes the edge from $V_{i}$ to each adjacent $V_{l}$, where $V_{l} \in C, \rho_{*}$ denotes the push forward of $\rho$, and where edges related by $\rho_{*}$ are associated with corresponding inputs in $V_{i}$ and $V_{j}$.

The definition above describes symmetry based on the system equations as in Equation 1; however, symmetry can be directly applied to the graphical representation by interchanging vertices with the same valence, while changing the edges using Equation 4 . The valence of a vertex is the number of edges that have the vertex an end-point or initial point. A digraph is said to be nonlinear distributed symmetric if the corresponding system remains invariant when one or more vertices are interchanged.

These definitions are illustrated by the following example. Consider the system

$$
\Sigma: \quad \dot{x}=g_{1}(x) u_{1}+\cdots+g_{7}(x) u_{7},
$$

described by

$\left[\begin{array}{c}\dot{x}_{1} \\ \dot{x}_{2} \\ \dot{x}_{3} \\ \dot{x}_{4} \\ \dot{x}_{5} \\ \dot{x}_{6} \\ \dot{x}_{7}\end{array}\right]=\left[\begin{array}{c}x_{1} \\ x_{2} \\ x_{3} \\ x_{4} \\ x_{5} \\ x_{6} \\ x_{7}\end{array}\right] u_{1}+\left[\begin{array}{c}x_{2} \\ 1 \\ \sin x_{2} \\ 0 \\ 0 \\ 0 \\ x_{2}\end{array}\right] u_{2}+\cdots+\left[\begin{array}{c}x_{7} \\ \sin x_{7} \\ 0 \\ 0 \\ 0 \\ x_{7} \\ 1\end{array}\right] u_{7}$

where $x_{i} \in V_{i}$ and each vertex has one associated input $u_{i}$ for $i=\{1, \ldots, 7\}$. (Note that in general each vertex can represent more than on state and associated with which could be more than one input). Note that, for example the $\sin \left(x_{2}\right)$ in the third slot in $g_{2}$ is represented in $\mathcal{G}_{\Sigma}$ by the edge directed from $V_{2}$ to $V_{3}$ and the $\sin \left(x_{7}\right)$ in the second slot of $g_{7}$ is represented in $\mathcal{G}_{\Sigma}$ by the edge directed from $V_{7}$ directed to $V_{2}$. These two edges map the states from the initial vertex to the tangent space of adjacent vertex (along the clockwise cycle) in the same way; hence, $E_{2,3}=\rho E_{7,2}$ if $\rho$ interchanges $V_{2}$ and $V_{7}$.

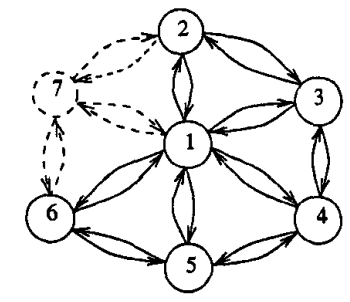

Figure 3. Removal of vertex $V_{7}$ and corresponding edges $E_{27}, E_{72}, E_{17}, E_{71}, E_{67}$, and $E_{76}$.

With the relation between a system and digraph formally defined, transformation between digraphs can now be defined. Two transformation are considered in this paper, contraction and expansion. System contraction is transformation between systems that reduces a system with $n$ subsystems and symmetry $S_{m}$ to a system with $n-1$ subsystems and symmetry $S_{m-1}$. Expansion maps a system with $n$ subsystems and symmetry $S_{m}$ to a system with $n+1$ subsystems and symmetry $S_{m+1}$.

Given a digraph, $\mathcal{G}_{\Sigma_{n}}=\left\{\mathbf{V}_{\mathbf{n}}, \mathbf{E}_{\mathbf{n}}\right\}$, contraction acts on both vertices and edges. Contraction deletes one vertex, $V_{j}$, and all edges connected to $V_{j}$, i.e., all edges of the form $E_{i, j}$ and $E_{j, i}$. In Figure 3, vertex $V_{7}$ is being removed from the example system along with all edges. The dashed lines in this figure represent elements that are being removed. New edges are added to the digraph as needed to maintain an $S_{m-1}$ symmetry. Let $V_{a_{1}}$ and $V_{a_{2}}$ denote vertices adjacent to the removed vertex, $V_{j}$. Without loss of generality, assume that $V_{a_{2}}$ precedes $V_{a_{2}}$ in the clockwise cycle, $C_{1}$, and $V_{a_{2}}$ precedes $V_{a_{1}}$ in the counter clockwise cycle, $C_{2}$. Edges connecting vertices $V_{a_{1}}$ and $V_{a_{2}}$ are created by applying the push forward of the symmetry operator, $\rho \in S_{m-1}$, on non-deleted edges such that for the vertices $V_{a_{1}}$ and $V_{a_{2}}$,

$$
\begin{aligned}
& E_{a_{1}, a_{2}}=\rho_{*} E_{j, l_{j+1}}, \\
& E_{a_{2}, a_{1}}=\rho_{*} E_{j, l_{j-1}},
\end{aligned}
$$

where $j$ is not adjacent to the removed vertex, $V_{j}$ and $E_{j, l_{j} \pm 1}$ denotes the edge from $V_{j}$ to each adjacent $V_{l}$, where $V_{l} \in C$. By construction, the new edges are symmetric with respect to an $S_{m-1}$ symmetry. Figure 4 shows the contracted digraph of the example system with dashed lines representing the edges that were added to maintain an $S_{5}$ symmetry.

Expansion of digraphs is the converse of contraction. Instead of removing a vertex, one is added such that symmetry is maintained. Graphically, this is done by deleting edges connecting two vertices from the orbit, if such edges exist. All other edges are left unchanged. 


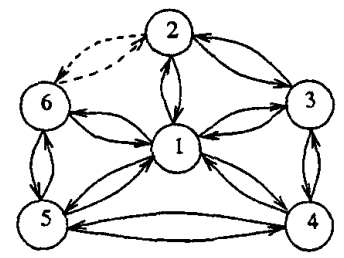

Figure 4. Addition of edges $E_{62}$ and $E_{26}$ to complete $S_{5}$ symmetry.

A copy of a vertex in the orbit is added to the digraph. Edges are added connecting the new vertex to all fixed vertices. Finally, edges connecting the new vertex to vertices in the orbit are added to maintain an $S_{m+1}$ symmetry. All new edges are copies of existing edges connecting similar orbits.

Expansion adds a complete subsystem to the system equations. This addition is equivalent to the addition of a vertex in the graphical representation. The structure of the added subsystem has the same structure of an existing subsystem in the symmetry orbit. Let $V_{c}$ represent the new vertex. The vertex $V_{c}$ must be related to an existing vertex, $V_{i} \in \mathbf{V}_{\mathbf{n}}$, by the symmetry operator, $\rho \in S_{m+1}$, i.e., $V_{c}=\rho V_{i}$. New edges are created by applying the push forward of the symmetry operator, $\rho \in S_{m-1}$, on non-deleted edges as follows. Assume that $V_{b_{1}}$ and $V_{b_{2}}$ are adjacent to the added vertex where $V_{b_{2}}$ precedes $V_{b_{2}}$ in the clockwise cycle, $C_{1}$, and $V_{b_{2}}$ precedes $V_{b_{1}}$ in the counter clockwise cycle, $C_{2}$, then

$$
\begin{array}{ll}
E_{b_{1}, c}=\rho_{*} E_{j, l_{j+1}}, & E_{c, b_{1}}=\rho_{*} E_{j, l_{j-1}}, \\
E_{b_{2}, c}=\rho_{*} E_{j, l_{j+1}}, & E_{c, b_{2}}=\rho_{*} E_{j, l_{j-1}},
\end{array}
$$

where $j$ is not adjacent to the added vertex, $V_{c}$ and $E_{j, l_{j} \pm 1}$ denotes the edge from $V_{j}$ to each adjacent $V_{l}$. Now, for each fixed vertex $V_{f}$

$$
\begin{aligned}
& E_{f, c}=\rho_{*} E_{f, j}, \\
& E_{c, f}=\rho_{*} E_{j, f},
\end{aligned}
$$

where $j$ is not adjacent to the added vertex. By construction, all new edges are symmetric with respect to an $S_{m+1}$ symmetry.

Now, define an equivalence class of control systems, $\bar{\Sigma}$ where for each $\Sigma_{n} \in \bar{\Sigma}, \Sigma_{n+1}$ and $\Sigma_{n-1}$ are equivalent to $\Sigma_{n}$ related to $\Sigma_{n}$ by the expansion and contraction constructions previously defined.

\section{Reduction and Controllability}

First, it is necessary define the term "controllable." Given an open set $W \subseteq M$, define $R^{W}\left(x_{0}, T\right)$ to be the set of states $x$ such that there exists $u:[0, T] \rightarrow \mathcal{U}$ that steers the control system from $x(0)=x_{0}$ to $x(T)=x_{f}$ and satisfies $x(t) \in W$ for $0 \leq t \leq T$, where $\mathcal{U}$ is the set of admissible controls. Define

$$
R^{W}\left(x_{0}, \leq T\right)=\bigcup_{0<\tau \leq T} R^{W}\left(x_{0}, \tau\right) .
$$

We will refer to $R^{W}\left(x_{0}, \leq T\right)$ as the set of states reachable up to time $T$.

Definition $3.1 A$ system is small time locally controllable ("STLC," or simply "controllable") if $R^{W}\left(x_{0}, \leq T\right)$ contains a neighborhood of $x_{0}$ for all neighborhoods $W$ of $x_{0}$ and $T>0$.

Let $\mathcal{C}$ denote the smallest subalgebra of $V^{\infty}(M)$ (the Lie algebra of smooth vector fields on a manifold $M$ whose product is the Lie bracket, $[\cdot, \cdot])$ that contains $g_{1}, \ldots, g_{m}$. If $\operatorname{dim}(\mathcal{C})=\operatorname{dim} M$ at a point $x$, then the system described by Equation 1 satisfies the Lie Algebra Rank Condition ("LARC") at $x$. The following is well known as "Chow's Theorem."

THEOREM 3.2 If the system described by Equation 1 satisfies the LARC at a point $x_{0}$ then it is STLC from $x_{0}$.

The following proposition is the main result in this paper.

Proposition 3.3 If any one member, $\Sigma_{n}$, of the equivalence class of symmetric distributed control systems, $\bar{\Sigma}$ is STLC, then all members of the equivalence class, $\Sigma_{i} \in \bar{\Sigma}$ where $i>n$ of symmetric distributed control systems are $S T L C$.

Proof: Assume that $\Sigma_{n} \in \bar{\Sigma}$, satisfies Chow's theorem. Partition the configuration manifold into sets of states corresponding to each node in the control system, i.e., let $M=\Pi_{i=1}^{n} V_{i}$. Let $\Sigma_{n}$ contain $n$ nodes with $m$ nodes in the $G$-orbit, and denote $\Delta_{i}$ to be the subdistribution of $\bar{\Delta}_{n}$ which spans the tangent space to the states associated with node $i$. Since $\Sigma_{n}$ is STLC,

$$
\bar{\Delta}_{n}=\sum_{i=1}^{m} \Delta_{i},
$$

where the sum of distributions is defined in a pointwise manner as in [3]. We will show that if $\Sigma_{n}$ is STLC, then $\Sigma_{n+1}$ is STLC, and then the result follows by induction.

For $\Sigma_{n+1}$, recall that the transformation from $\Sigma_{n}$ to it was that a vertex (submanifold), $V_{j}$, was added to the $G$-orbit. If edges connected the elements of the $G$ orbit, one edge was deleted, and copies of existing edges were added to maintain symmetry. We will consider separately the fixed nodes, nodes in the $\mathcal{G}$-orbit not $V_{j}$ and not adjacent to $V_{j}$, the nodes adjacent to $V_{j}$ and $V_{j}$ itself. 
For nodes in the orbit not adjacent to $V_{j}$, denoted by $\Delta_{i}$, let the collection of vector fields $\left\{X_{1}, \ldots, X_{q}\right\} \in$ $\bar{\Delta}_{n}$ span $\Delta_{i}$. Then in $\Sigma_{n+1}$ the same set of vector fields will span $\Delta_{i}$ in $\Sigma_{n+1}$ where the vector fields, $X_{i}$ are defined relative (in position) to $V_{i}$. If an adjacent node, say $V_{k}$, is a fixed node, i.e., not in the $G$-orbit, then $\Delta_{k}$ will still span $T V_{k}$ because vector fields have only been added to that node, and the existing vector fields are unchanged. For $V_{j}$ and the adjacent vertices, by symmetry $\exists \rho \in S_{m+1}$ such that $V_{j}=\rho V_{l}$, where $V_{l}$ is a nonadjacent node. Since $\Delta_{l}$ spans $T V_{l}$, and the system in invariant with respect to the group action, $\Delta_{j}$ must span $T V_{j}$. Similarly, for the vertices adjacent to $V_{j}, \exists \nu \in S_{m+1}$ such that $\nu V_{l}$ is mapped to them, which shows that $\Delta$ for the adjacent nodes in the $G$-orbit must span their tangent spaces. Since $\exists \Delta_{i}$ such that $\operatorname{span} \Delta_{i}=T V_{i}, \Delta_{n+1}=\sum_{i=1}^{n+1} \Delta_{i}$ spans $T M=\prod_{i=1}^{n+1} T V_{i}$. Since $M$ is a manifold, by Frobenius' theorem, $\Delta_{m+1}$ is involutive. Therefore, $\bar{\Delta}_{m+1}$ is full rank, and by Chow's theorem, the system is STLC.

This proposition only provides a sufficient condition for controllability of larger symmetric systems if a smaller equivalent system is controllable. The necessary condition requires further assumptions regarding the largest degree of Lie bracket necessary for $\bar{\Delta}$ to be involutive. Detailed computations illustrate that states from one node can "propagate" to affect other nodes via Lie brackets. In fact, they can "propagate" one node for each order of Lie bracket. We note that even though this proposition does not provide the necessary condition for controllability of a larger system, the sufficient condition is of greater engineering utility due to the fact that it can be used to determine controllability of a larger system by analyzing a smaller system.

\section{Cooperating Robotic Systems}

A simple robotic example is given in this section to demonstrate the utility of the results presented in this paper. Suppose we have a group of 5 mobile robots, where an individual mobile robot is described by

$$
\left[\begin{array}{c}
\dot{x} \\
\dot{y} \\
\dot{\theta}
\end{array}\right]=\left[\begin{array}{c}
\cos \theta \\
\sin \theta \\
0
\end{array}\right] u_{1}+\left[\begin{array}{l}
0 \\
0 \\
1
\end{array}\right] u_{2}
$$

where $u_{1}$ is the linear velocity input and $u_{2}$ is the angular velocity input from [9]. We will control the the group using a leader/follower method where robot one is the leader. The leading robot controls the angular position of the entire group. The following robots have individual control of their respective linear speed, $u_{1}$, but not their direction. To ensure following robots do not overtake the leading robot, the linear velocity in-

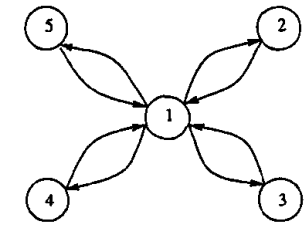

Figure 5. A digraph of five cooperating mobile robots.

put of the following robots directly affects the linear speed of the leading robot. After relabeling the states, as $x_{1}=x, x_{2}=y$, and $x_{3}=\theta$, the system equations for this configuration are

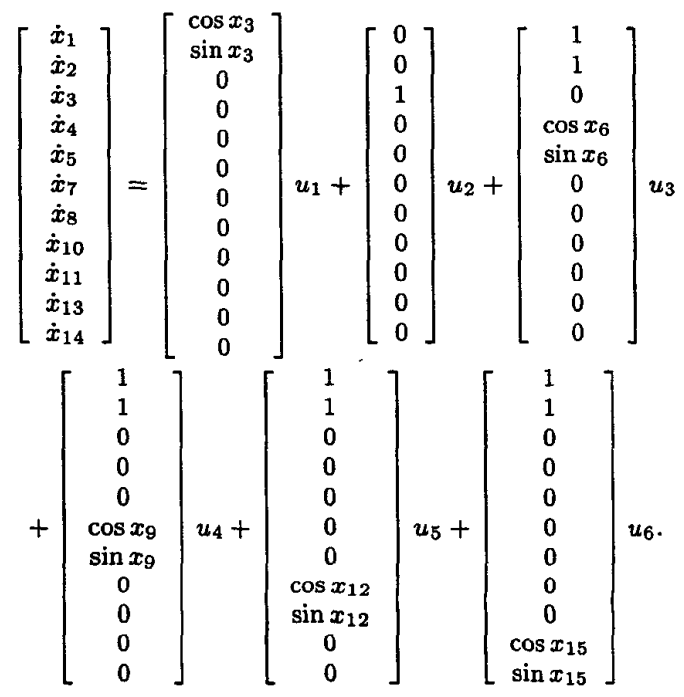

where $x_{4}$ represents the $x$ position of robot $2, x_{5}$ represent the $y$ position of robot 2 , etc. States $x_{6}, x_{9}, x_{12}$, and $x_{15}$ have be removed because they are all equal to $x_{3}$. A graph of this system is shown in Figure 5 .

Determining the controllability of this system and perhaps even larger systems can be tedious. We will use Proposition 3.3 to determine controllability of the complete system on a reduced order system. Using the contraction transformation described in Section 2, the example system can be reduced to a system of three vertices. Figure 6 displays the graph of the reduced system.

The equation corresponding to the reduced graph is

$$
r l\left[\begin{array}{c}
\dot{x}_{1} \\
\dot{x}_{2} \\
\dot{x}_{3} \\
\dot{x}_{4} \\
\dot{x}_{5} \\
\dot{x}_{7} \\
\dot{x}_{8}
\end{array}\right]=\left[\begin{array}{c}
\cos x_{3} \\
\sin x_{3} \\
0 \\
0 \\
0 \\
0 \\
0
\end{array}\right] u_{1}+\left[\begin{array}{l}
0 \\
0 \\
1 \\
0 \\
0 \\
0 \\
0
\end{array}\right] u_{2}
$$




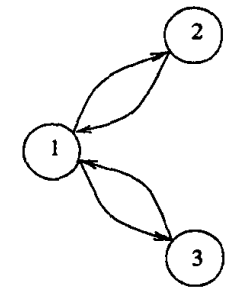

Figure 6. The three vertex reduced digraph for the example system.

$$
+\left[\begin{array}{c}
1 \\
1 \\
0 \\
\cos x_{6} \\
\sin x_{6} \\
0 \\
0
\end{array}\right] u_{3}+\left[\begin{array}{c}
1 \\
1 \\
0 \\
0 \\
0 \\
\cos x_{9} \\
\sin x_{9}
\end{array}\right] u_{4} \text {. }
$$

Let $g_{1}, g_{2}, g_{3}$, and $g_{4}$ be the vector fields given in the reduced equation. Taking Lie brackets

$$
\begin{gathered}
g_{5}=\left[g_{1}, g_{2}\right]=\left[\begin{array}{c}
\sin x_{3} \\
-\cos x_{3} \\
0 \\
0 \\
0 \\
0 \\
0
\end{array}\right], \quad g_{6}=\left[g_{2}, g_{3}\right]=\left[\begin{array}{c}
0 \\
0 \\
0 \\
-\sin x_{9} \\
\cos x_{9} \\
0 \\
0
\end{array}\right], \\
\text { and } g_{7}=\left[g_{2}, g_{4}\right]=\left[\begin{array}{c}
0 \\
0 \\
0 \\
0 \\
0 \\
-\sin x_{9} \\
\cos x_{9}
\end{array}\right],
\end{gathered}
$$

we find that the distribution spanned by the vector fields $g_{1}, g_{2}, g_{3}, g_{4}, g_{5}, g_{6}$, and $g_{7}$ is full rank. Therefore, by Chow's Theorem, the $x$ and $y$ positions of the reduced system are controllable. By Proposition 3.3, the full system of mobile robots is also controllable. Furthermore, the results show that the entire equivalence class of systems is controllable with respect to the $x$ and $y$ positions.

\section{Conclusions and Future Work}

In this paper, we have considered controllability of distributed systems. The main result was proving that controllability of large scale symmetric nonlinear systems can be determined from the controllability of a reduced order system. In fact, the result shows that controllability of an entire equivalence class of systems can be determined by checking the controllability of only one of its members.

Current efforts are being directed toward formulation the necessary condition for the larger-scale system to be controllable. Furthermore, this paper has presented distributed controllability for driftless nonlinear systems. The next step will be to extend this work to systems with drift, i.e, systems of the form

$$
\Sigma: \quad \dot{x}=f(x)+\sum_{i=1}^{m} g_{j}(x) u_{j} .
$$

In the future an extension of this work to multiple orbit systems with more complex interactions will also be considered.

\section{References}

[1] Anthony M. Bloch, P.S. Krishnaprasad, Jerrold E. Marsden, and Richard M. Murray. Nonholonomic mechanical systems with symmetry. Arch. Rational Mech. Anal., 136:21-99, 1996.

[2] W.L. Chow. Über systeme von linearen partiellen differentialgleichungen erster ordinung. Math Ann., 117:98-105, 1939.

[3] Alberto Isidori. Nonlinear Control Systems. SpringerVerlag, second edition, 1989.

[4] Jair Koiller. Reduction of some classical non-holonomic systems with symmetry. Archive for Rational Mechanics and Analysis, 118(2):113-148, 1992.

[5] Jerrold E. Marsden, Richard Montgomery, and Tudor S. Ratiu. Reduction, symmetry and phases in mechanics. Mem. Amer. Math. Soc., 436, 1990.

[6] Jerrold E. Marsden, Richard Montgomery, and Tudor S. Ratiu. Redumction, symmerty and phases in mechanics. Memoirs of the Americal Mathematical Society, 88:436, 1990.

[7] Jerrold E. Marsden and J. Scheurle. Lagrangian reduction and the double spherical pendulum. ZAMP, 44:17-43, 1993.

[8] Jerrold E. Marsden and J. Scheurle. The reduced eulerlagrange equations. Fields Institute Communications, 1:139-164, 1993.

[9] Shankar Sastry. Nonlinear Systems: Analysis, Stability, and Control, chapter 11. Springer, 1999.

[10] Hector J. Sussmann. A general theorem on local controllability. Siam J. Control and Optimization, 25(1):158-194, 1987.

[11] Reiko Tanaka and Kazuo Murota. Quantitative analysis for controllability of symmetric control systems. Int. $J$. Control, 73(3):254-264, 2000.

[12] Reiko Tanaka, Seiichi Shin, and Noboru Sebe. Controllability of autonomous decentralized systems. In Symposium on Emerging Technologies \& Factory Automation, pages 265-272. IEEE, 1994.

[13] Reiko Tanata and Seiichi Shin. Fearful symmetry in system structure. In Third International Symposium on $A u-$ tonomous Decentralized Systems, pages 137-146. IEEE, 1997.

[14] Arjan van der Schaf. Partial symmetries for nonlinear systems. Mathematical Systems Theory, 18(1):79-96, 1985.

[15] Arjan van der Schaft. Symmetries and conservation laws for hamiltonian systems with inputs and outputs: A generalization of noether's theorem. Systems \& Control Letters, $1(2), 1981$. 